\title{
Maximum entropy perception-action space: a Bayesian model of eye movement selection
}

\author{
Francis Colas ${ }^{*, \dagger}$, Pierre Bessière ${ }^{* *}$ and Benoît Girard ${ }^{\ddagger} \dagger$ \\ ${ }^{*}$ ASL - ETH Zürich, Tannenstrasse 3, 8092 Zürich Switzerland \\ ${ }^{\dagger}$ LPPA - CNRS/Collège de France, 11 place Marcelin Berthelot, 75005 Paris, France \\ ${ }^{* *}$ LIG - CNRS, 655 avenue de l'Europe, 38334 Saint Ismier, France \\ ${ }^{\ddagger} I S I R-C N R S / U P M C$, 4 place Jussieu, 75005 Paris, France
}

\begin{abstract}
In this article, we investigate the issue of the selection of eye movements in a free-eye Multiple Object Tracking task. We propose a Bayesian model of retinotopic maps with a complex logarithmic mapping. This model is structured in two parts: a representation of the visual scene, and a decision model based on the representation. We compare different decision models based on different features of the representation and we show that taking into account uncertainty helps predict the eye movements of subjects recorded in a psychophysics experiment. Finally, based on experimental data, we postulate that the complex logarithmic mapping has a functional relevance, as the density of objects in this space in more uniform than expected. This may indicate that the representation space and control strategies are such that the object density is of maximum entropy.
\end{abstract}

Keywords: Bayesian modelling, eye movements, retinotopic maps.

\section{INTRODUCTION}

In this paper, we study the evaluation of uncertainty for the selection processes related to active perception. Uncertainty is the consequence of both the inverse nature of perception and the necessary incompleteness of the models. We choose to handle uncertainty in a reasoning paradigm: the Bayesian Programming framework [1]. The experimental basis is a free-eye version of the standard Multiple Object Tracking (MOT) paradigm [2]. In this experiment, the subjects are presented with a number of moving identical objects. Some of these objects are designated at the beginning of each trial to be targets while the others are distractors. When the objects move, no cue can help distinguishing the targets. The task is to point out the targets at the end of the trial, which requires to track during the trial.

The Bayesian models we design compute a sequence of probability distributions over the next eye movement to perform, based on a sequence of observations of objects in the visual field. They are inspired by the anatomy and electrophysiology of eye-movement selection related brain regions. These regions (fig. 1), the superior colliculus (SC), the frontal eye fields (FEF), and the lateral bank in the intraparietal sulcus (LIP) share a number of properties. They all receive information concerning the position of points of interest in the visual field (visual activity), memorize them (delay activity) and can generate movements towards them (motor activity) $[4,5,6]$.

These positions are encoded by topographically organized cells, with receptive/motor fields defined in retinotopic reference frames. In the SC of primates, these maps have a complex logarithmic mapping [7,8], shown in fig. 2 by the blue lines (plain lines: iso- 


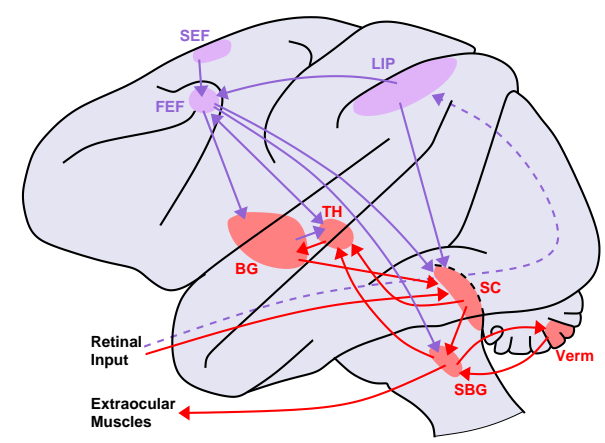

FIGURE 1. Premotor and motor circuitry shared by saccade and smooth pursuit movements (Macaque monkey). In red, short subcortical loop, in purple, long cortical loop. The dashed arrow stands for the cortical pathway including notably the lateral geniculate nucleus and the visual cortex. BG: basal ganglia, FEF: frontal eye fields, LIP: lateral bank of the intraparietal sulcus, SBG: saccade burst generators, SC: superior colliculus, SEF: supplementary eye fields, TH: thalamus, Verm: cerebellar vermis. Adapted from [3].

eccentricities; dotted lines: iso-directions). Concerning the FEF, the eccentricity of the position vector is encoded logarithmically [9], however the encoding of direction is not well understood yet. Finally, the structure of the LIP maps is still to be deciphered, but a continuous topographical organization seems to exist, with an over-representation of the central visual field [10]. We thus use the primate SC maps geometry in our models, with the assumptions that human SC and cortical maps probably have a similar geometry.

The spatial working memory-related neurons in SC [11], FEF [12] and LIP [13] are capable of dynamic remapping. They can be activated by a memory of the position of a target, even if the target was not in the cell's receptive field at the time of presentation. They behave as if they were part of a retinotopic memory map, where a remapping mechanism would allow the displacement of the memorized activity when an eye movement is performed. We include this remapping capability in our models.

We first present the structure of our models, then we compare their movement predictions with recorded human movements. Finally we show that explicitely using uncertainty improves the quality of the prediction.

\section{MODEL}

Our model has two stages: a representation of the visual field and the decision process of the next eye movement.

\section{Representation}

The representation model is a dynamic retinotopic map of the objects in the visual field. This representation is structured in two successive layers: the occupancy of the visual field, and a memory of the position of each target. 
Occupancy of the visual field. The first part is structured like an occupancy grid, a recursive Bayesian filter introduced for obstacle representation in mobile robotics [14]. The environment is discretized into a regular grid $\mathscr{G}$ (with the logcomplex mapping) and we define a binary variable $O c c_{c}^{t}$ in each cell $c$ and for each time $t$ that states whether or not there is an object in the corresponding location in the visual field. The input is introduced as a set of binary variables $\mathrm{Obs}_{c}^{t}$. The observation and occupancy of each cell are linked by a probabilistic relation $P\left(O b s_{c}^{t} \mid O c c_{c}^{t}\right)$ for the observation of a cell's occupancy. As for all subsequent probability distributions that appear in our models, we give this probability distribution a parametrical form whose parameters we learn for part of the experimental data.

The remapping capability of this model relies on the current displacement $M v t^{t}$ and the distribution $P\left(O c c_{c}^{t} \mid O c c^{t-1} M v t^{t}\right)$ that transfers the occupancy associated to antecedent cells to the corresponding present cell with an additional uncertainty factor.

Due to the high dimensionality of this representation space, we approximate the inference over the whole grid by a set of inferences for each cell $c$ that depend only on a subset $\mathscr{A}(c)$ of antecedent cells $c^{\prime}$ for the current eye movement. Thus the update of the knowledge on occupancy in our model is recursively computed as follows:

$$
P\left(O c c_{c}^{t} \mid O b s^{1: t} M v t^{1: t}\right) \propto P\left(O b s_{c}^{t} \mid O c c_{c}^{t}\right) \sum_{O c c_{\mathscr{A}(c)}^{t-1}}\left[\begin{array}{l}
P\left(O c c_{c}^{t} \mid M v t^{t} O c c_{\mathscr{A}(c)}^{t-1}\right) \\
\times \prod_{c^{\prime}} P\left(O c c_{c^{\prime}}^{t-1} \mid O b s^{1: t-1} M v t^{1: t-1}\right)
\end{array}\right.
$$

Position of the targets. To introduce the discrimination between targets and distractors, we add a set of variables $T g t_{i}^{t}$ that represent the location of each target $i$ at each time $t$. We also include remapping capability for the targets so that an eye movement $M v t^{t}$ updates the distribution on $T g t_{i}^{t}$. This is done in a dynamic model $P\left(T g t_{i}^{t} \mid T g t_{i}^{t-1} O c c^{t} M v t^{t}\right)$ similar to the dynamic model of occupancy.

In addition to question 1 , the knowledge over the targets is computed as follows:

$$
P\left(T g t_{i}^{t} \mid O b s^{1: t} M v t^{1: t}\right) \propto \sum_{T g t_{i}^{t-1}}\left[\begin{array}{l}
P\left(T g t_{i}^{t-1} \mid O b s^{1: t-1} M v t^{1: t-1}\right) \\
\times \sum_{O c c^{t}}\left[\begin{array}{l}
P\left(O c c^{t} \mid O b s^{1: t} M v t^{1: t}\right) \\
\times P\left(T g t_{i}^{t} \mid T g t_{i}^{t-1} O c c^{t} M v t^{t}\right)
\end{array}\right)
\end{array}\right.
$$

where the summation over the whole grid can be approximated, by separating the cells.

Equations 1 and 2 represent the current knowledge about the visual scene that can be inferred from the past observations and movements and the hypotheses of our model.

\section{Decision}

Based on this knowledge, we propose models that determine where to look next. We make the hypothesis that the representation model exposed above is useful for producing eye movements.

The main hypothesis is that uncertainty explicitly taken into account can help in the decision of eye movement. Thus we compare one model that does not take explicitly into account the uncertainty, target model, with one that does, uncertainty model. 
Constant model. This model is a baseline for the other two. It is defined as the best static probabilistic distribution $P(M o t)$ that can account for the experimental eye movement. In this distribution, the probability for a given eye movement is equal to its experimental frequency. Thus we learned this distribution from our experimental data.

Target model. This second model determines its eye movements based on the location of the targets. It is a Bayesian fusion model with each target considered as the location where to look. It uses an inverse model $P\left(T g t_{i}^{t} \mid M o t^{t}\right)$ that states that at time $t$ the location of the target $T g t_{i}^{t}$ is probably near the eye movement $M_{o t} t^{t}$ with a Gaussian distribution. Moreover, the prior distribution on the eye movement is taken from the constant model. Therefore, this target model refines the eye movement distribution with the influence of each targets.

As the exact locations of the targets are not known, this model takes into account the estimation from question 2 in the fusion. The actual eye movement distribution can be computed using the following expression:

$$
P\left(M^{t} t^{t} \mid O b s^{1: t} M v t^{1: t}\right) \propto P(M o t) \prod_{i=1}^{N} \sum_{T_{g} t_{i}^{t}} P\left(T g t_{i}^{t} \mid O b s^{1: t} M v t^{1: t}\right) P\left(T g t_{i}^{t} \mid M o t^{t}\right)
$$

Uncertainty model. The behaviour of the previous model is influenced by uncertainty insofar as the incentive to look near a given target is higher for a more certain location of this target. As for any Bayesian model, uncertainty is handled as part of the inference mechanism: as a means to describe knowledge.

In this third model, we propose to include uncertainty as a variable to reason about: as the knowledge to be described. The rationale is simply that it is more efficient to gather information when and where it lacks: that is when and where there is more uncertainty.

Therefore, we introduce a new set of variables $I_{c}^{t}$ representing an uncertainty index at cell $c$ at time $t$. For this implementation, we choose to specify this uncertainty index as the probability distribution of occupancy in this cell. The nearer this probability is to $\frac{1}{2}$ the higher the uncertainty and the higher the probability to look there. In the end, this model computes the posterior probability distribution on next eye movement using the following expression:

$$
P\left(M o t^{t} \mid O b s^{1: t} M v t^{1: t} I^{1: t}\right) \propto P\left(M o t^{t} \mid O b s^{1: t} M v t^{1: t}\right) P\left(I_{\left[c=M o t^{t}\right]}^{t} \mid M o t^{t}\right)
$$

with $I_{c}^{t}=P\left(O c c_{c}^{t} \mid O b s^{1: t} M v t^{1: t}\right)$ (equation 1) and $P\left(I_{c}^{t} \mid M o t_{t}\right)$ a beta distribution with equal parameters (with maximum at $\frac{1}{2}$ ).

This model filters the eye movement distribution computed by the second model, in order to enhance the probability distribution in the locations of high uncertainty.

\section{RESULTS}

As shown in figure 2, these models produce a probability distribution at each time step which is, except for the constant model, heavily dependent on the past observations and movements in the retinocentered reference frame. Therefore we first defined an appropriate tool to compare these model. Then we present the results of our models according to this evaluation method. 


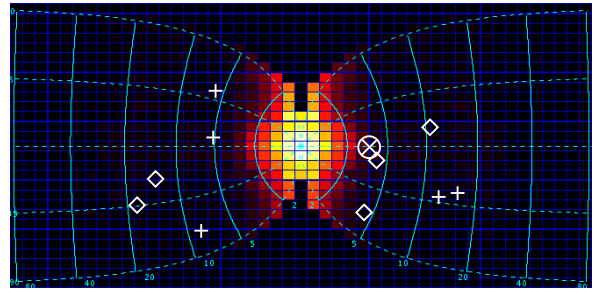

(a)

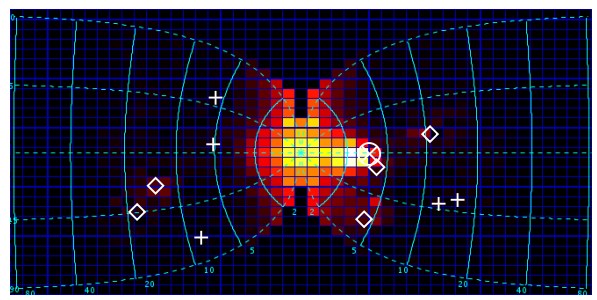

(c)

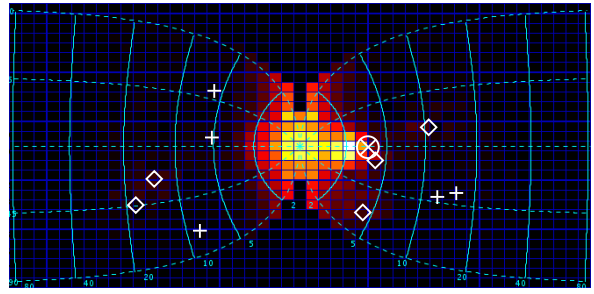

(b)

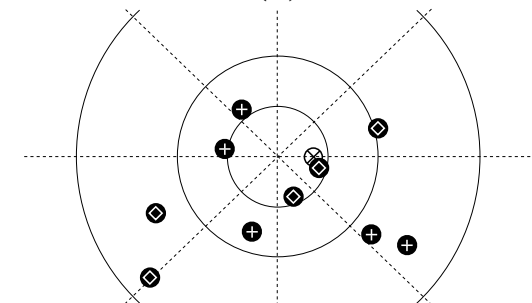

(d)

FIGURE 2. Example of probability distributions computed by each model in the same configuration. Panel (a) is the distribution of constant model. Panel (b) shows the probability distribution for the target model that shows a preference for the targets. Panel (c) shows the probability distribution for the uncertainty model that highlights some of the targets. Bottom panel shows the position of the targets (magenta) and objects (red) in the visual field. Reprinted from [15].

\section{Comparison method}

The generic Bayesian method to compare models (or parameters, that is formally the same issue) is to assess a prior probability distribution over the models, compute the likelihood of each model in view of the data, and use Bayes rule to obtain a probability distribution over the models: $P($ Model $\mid$ Data $) \propto P($ Model $) \times P($ Data $\mid$ Model $)$.

As deciding on priors is sometimes an arbitrary matter and this prior may have a negligible influence with a growing number of data points, a common approximation is simply comparing the likelihood of the models. Choosing the model with the highest likelihood is dubbed as maximum likelihood estimation.

As the decision models compute a probability distribution, we can compute, for each model at each time step, the probability of the actual eye movements recorded from subjects, as well as the probability of the whole set of recordings. In order not to have a measure that tends to zero as the number of trials increase, we choose the geometric mean of the likelihood across trials, as it tends to be independent on the number of trials.

\section{Tests}

The data set is gathered from 11 subjects with 110 trials each for a total of 1210 trials (see [16] for details). Each trial was discretized in time in 24 observations for a grand total of 29040 data points. Part of the data set (124 random trials) was used to determine the 9 parameters of the model and the results are computed on the remaining 1089 trials.

Table 1 presents the results of our three decision models for this data set. It shows that 
TABLE 1. Ratio of the measures for pair of models.

\begin{tabular}{|c|c|c|c|}
\hline Ratio & Constant & Target & Uncertainty \\
\hline Constant & 1 & 280 & 320 \\
\hline Target & $3.5 \times 10^{-3}$ & 1 & 1.14 \\
\hline Uncertainty & $3.1 \times 10^{-3}$ & 0.87 & 1 \\
\hline
\end{tabular}

the model that generates motion with the empiric probability distribution but without the representation layer is far less probable than the other two (by respectively a factor 280 and 320). The representation layer is thus useful in deciding the next eye movement.

Table 1 further shows that the model taking explicitly into account uncertainty is better than the model that does not by $14 \%$. This is in favor of our hypothesis that taking explicitly into account uncertainty is helpful in deciding the next eye movement.

It should be noted that the choice of the geometric mean prevents the ratio of our models to raise exponentially as the number of trials grows. In our case, the likelihood ratio between the model with explicit uncertainty and the one without is $4.9 \times 10^{63}$. With half the trials, this likelihood ratio is the square root, that is only $7.0 \times 10^{31}$. We preferred presenting the results with a measure independent of the number of trials.

Moreover, this model is designed to study the criteria of eye movement selection and not to solve the task autonomously. Nevertheless, we can add simple algorithms to decide the eye movement at each time step and to point out the targets at the end of the trial, based on the state of the representation layer. A naive implementation yielded a chance level performance. We did not have the opportunity to delve deeper into this question, as this is not the aim of our model, but this result could be improved by a more accurate target layer.

\section{Complex Logarithmic Mapping}

One feature of the model is the choice of the complex logarithmic mapping of the visual field for the representation space. This choice is motivated by the organization of the retinotopic maps, for example the SC but a model with the same principles with a representation in the visual field would have the same global results. However, the distribution of objects with respect to the gaze position, relies heavily on the mapping. For example, a distribution uniform with the log complex mapping would yield a sharply peaked distribution in the visual space, whereas a uniform distribution in the visual space would yield a lower density in the middle (as more representation space is dedicated to less visual space) and a higher density in the periphery.

In our experiment, the objects trajectory cover the screen uniformly. In one additional experiment condition (see [16]) the subjects were required to fixate their gaze. In this condition, the objects are uniformly distributed on the visual field. Top row of figure 3 displays the experimental object distribution in fixed eyes condition in both the visual space (left) and using the complex logarithmic mapping (right). We can check that the distribution is uniform, except from a slight overrepresentation of the border and corners due to the way the objects bounce on this limit. In the complex logarithmic mapping, 

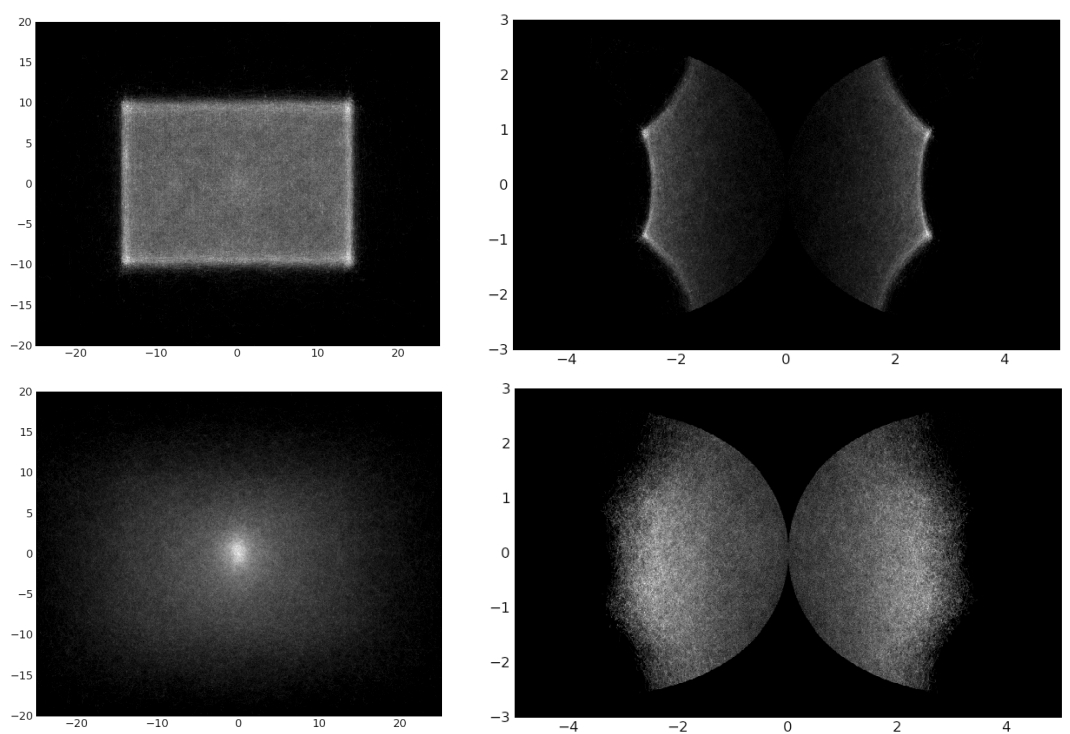

FIGURE 3. Object distribution. Top row, in fixed eye condition; bottom row in free eye condition. Left column in visual space; right column in complex logarithmic mapping.

this translate to a distribution nearly empty in the middle as expected.

On the other hand, the bottom row the figure 3 shows the object distribution in free eyes condition. As above, the left graph shows the distribution in visual space and the right graph shows the distribution in complex logarithmic space. In visual space, we see that the object density is higher near the center of the visual field as subjects tend both to follow targets with smooth pursuit and fixate in the neighborhood of objects. On the other hand, bottom right graph in figure 3, shows that the distribution of object in the complex logarithmic retinocentric space is nearly uniform. This is a property that is specific to the movement selection strategy and it is surprising as an arbitrary strategy looking at the targets or in their middle wouldn't necessarily yield such a distribution.

This shows that the eye movement selection strategy and the representation space are such that the distribution of salient objects in the representation space relative to the eye position is relatively uniform. From the point of view of information theory, this is the most efficient combination, as it maximizes the bandwith of the representation.

\section{CONCLUSION AND DISCUSSION}

As a conclusion, we propose a Bayesian model with two parts: a representation of the visual scene, and a decision model based on this representation. The representation both tracks the occupancy of the visual scene as well as the locations of the targets.

Based on this representation, we tested several decision models and we have shown that the model that takes explicitly into account the uncertainty better fitted the eye movements recorded from subjects participating a psychophysics experiment.

The difference between the target model and the uncertainty model, on the other hand is due to the filtering of the eye movements distribution from the target model 
by the uncertainty. The difference is less important than for the constant model as the uncertainty associated to the targets are often similar (isolated targets with comparable movement profiles). It could be interesting to enrich the stimulus in order to manipulate uncertainty more precisely.

Finally, the representation space is inspired by the geometry of the retinotopic brain areas which present a complex logarithmic mapping with the visual field. Analyses of the experimental eye movements of the subjects show that their selection is such that the distribution of the objects in this complex logarithmic mapping is nearly uniform.

\section{ACKNOWLEDGEMENTS}

This work was supported by the European Project BACS FP6-IST-027140. The authors thank Thomas Tanner, Luiz Canto-Pereira, and Heinrich Bülthoff for the experimental results and Fabien Flacher and Julien Diard for their help.

\section{REFERENCES}

1. Olivier Lebeltel, Pierre Bessière, Julien Diard, and Emmanuel Mazer. Bayesian robots programming. Autonomous Robots, 16(1):49-79, 2004.

2. Z.W. Pylyshyn and R.W. Storm. Tracking multiple independent targets: evidence for a parallel tracking mechanism. Spatial Vision, 3(3):1-19, 1988.

3. R.J. Krauzlis. Recasting the Smooth Pursuit Eye Movement System. Journal of Neurophysiology, 91(2):591-603, 2004.

4. A.K. Moschovakis, C.A. Scudder, and S.M. Highstein. The microscopic anatomy and physiology of the mammalian saccadic system. Prog Neurobiol, 50:133-254, 1996.

5. R.H. Wurtz, M.A. Sommer, M. Paré, and S. Ferraina. Signal transformation from cerebral cortex to superior colliculus for the generation of saccades. Vision Res, 41:3399-3412, 2001.

6. C.A. Scudder, C.R.S. Kaneko, and A.F. Fuchs. The brainstem burst generator for saccadic eye movements. A modern synthesis. Exp Brain Res, 142:439-462, 2002.

7. D.A. Robinson. Eye movements evoked by collicular stimulation in the alert monkey. Vision Res, 12:1795-1808, 1972.

8. F.P. Ottes, van Gisbergen J.A., and J.J. Eggermont. Visuomotor fields of the superior colliculus: a quantitative model. Vision Res, 26(6):857-873, 1986.

9. M.A. Sommer and R.H. Wurtz. Composition and topographic organization of signals sent from the frontal eye fields to the superior colliculus. Journal of Neurophysiology, 83:1979-2001, 2000.

10. S. Ben Hamed, J.-R. Duhamel, F. Bremmer, and W. Graf. Representation of the visual field in the lateral intraparietal area of macaque monkeys: a quantitative receptive field analysis. Experimental Brain Research, 140:127-144, 2001.

11. L.E. Mays and D.L. Sparks. Dissociation of visual and saccade-related responses in superior colliculus neurons. J Neurophysiol, 43(1):207-232, 1980.

12. M.E. Goldberg and C.J. Bruce. Primate frontal eye fields. III. maintenance of a spatially accurate saccade signal. Journal of Neurophysiology, 64(2):489-508, 1990.

13. J.W. Gnadt and R.A. Andersen. Memory related motor planning activity in the posterior arietal cortex of the macaque. Experimental Brain Research, 70(1):216-220, 1988.

14. A. Elfes. Occupancy grids: a probabilistic framework for robot perception and navigation. $\mathrm{PhD}$ thesis, Pittsburgh, PA, USA, 1989.

15. F. Colas, F. Flacher, T. Tanner, P. Bessière, and B. Girard. Bayesian models of eye movement selection with retinotopic maps. Biological Cybernetics, 100(3):203-14, 2009.

16. T.G. Tanner, L.H. Canto-Pereira, and H.H. Bülthoff. Free vs. constrained gaze in a multiple-objecttracking-paradigm. In 30th European Conference on Visual Perception, Arezzo, Italy, August 2007. 